\title{
Developing patient safety in dentistry
}
IN BRIEF
- Discusses the history of patient safety initiatives in healthcare and dentistry.
- Describes strategies that can be applied to identify patient safety issues in dentistry.
- Emphasises the importance of both process and cultural factors in developing a safer healthcare environment.

M. N. Pemberton ${ }^{1}$

\begin{abstract}
Patient safety has always been important and is a source of public concern. Recent high profile scandals and subsequent reports, such as the Francis report into the failings at Mid Staffordshire, have raised those concerns even higher. Mortality and significant morbidity associated with the practice of medicine has led to many strategies to help improve patient safety, however, with its lack of associated mortality and lower associated morbidity, dentistry has been slower at systematically considering how patient safety can be improved. Recently, several organisations, researchers and clinicians have discussed the need for a patient safety culture in dentistry. Strategies are available to help improve patient safety in healthcare and deserve further consideration in dentistry.
\end{abstract}

'The dental profession is committed to providing safe dental care, which is necessary for ensuring good general health, and aims to minimise risks and establish an open culture of patient safety, in which practitioners can learn from their own and others' experience.'

Patient safety and quality are high profile in healthcare at the moment. The landmark Francis report into what went wrong at Mid Staffordshire NHS Foundation Trust highlighted the risk to patient safety when the prevailing culture does not value quality of care as the most important factor in managing patients. ${ }^{2}$ The subsequent report on patient safety in the NHS, led by Don Berwick, stated as the first line of its executive summary: 'Place the quality of patient care, especially patient safety, above all other aims. ${ }^{3}$ As a profession, dentistry has been slower at joining our medical colleagues in systematically considering how patient safety can be improved. ${ }^{4,5}$ While the overall morbidity associated with dentistry is less than medicine, important challenges still remain such as can we reduce the risk of taking out the wrong tooth or can we reduce the risk of failing to identify an oral cancer?

${ }^{1}$ Clinical Head of Division, University Dental Hospital of Manchester, Central Manchester University Hospitals NHS Foundation Trust, Higher Cambridge Street, Manchester, M15 6FH

Correspondence to: Dr Michael N. Pemberton

Email: michael.n.pemberton@manchester.ac.uk

\section{Refereed Paper}

Accepted 19 June 2014

DOI: $10.1038 /$ sj.bdj.2014.856

${ }^{\circ}$ British Dental Journal 2014; 217: 335-337
At the University Dental Hospital of Manchester we have been considering how we can improve patient safety in our hospital and we have developed various initiatives in an attempt to do this. These include development of a clinical effectiveness dashboard, the development of checklists to use in a dental setting and a dental correct site surgery policy for use in an out-patient setting. These initiatives have been developed in a secondary care setting but are of relevance in the development of patient safety initiatives in other branches of dentistry.

This article draws upon some of the developments in thinking about patient safety that have occurred in medicine and briefly outlines some of the principles involved and how this work may be developed in dentistry.

It is wrong to think that patient safety has not been a consideration for all clinical practitioners for many years, although systematic consideration of how safety issues can be identified has been a more recent development. Formal enquiry into patient safety is not new, however, and patient safety initiatives have occurred throughout the last century. In 1952 the British Government established the Confidential Enquiry into Maternal Deaths to learn lessons from such tragic events. This was followed by the similar enquiry schemes into peri-operative deaths and into suicides/ homicides under the mental health services. In 1963, following the awareness of serious birth defects having been caused by the drug thalidomide, the Safety in Drugs committee was established. This has subsequently developed into the current Medicines and Healthcare Products Regulatory Authority (MHRA). In 1970, following various public enquiries into hospital failings, the Hospital Advisory Service was set up. This has since evolved into the Care Quality Commission (CQC). The 1970s and 1980s saw a growing culture of litigation with the office of the Health Service Ombudsman established to learn from complaints. In the 1990s, further inquiries into failures at Alder Hey Hospital and the Bristol paediatric cardiac service occurred while the increase in litigation led to the NHS litigation authority being set up.

Meanwhile, in 1991, an important study was published that highlighted to the medical profession the risks of error in medical care over a much wider range of patient groups than previously considered. ${ }^{6}$ This study reviewed more than 30,000 patient hospital records of 51 acute care, non-psychiatric hospitals in New York State. The records were studied for the extent of serious medical injury caused by medical treatment. The paper reported that 3.7\% of patients had an adverse event or injury caused by treatment, of which nearly two thirds were caused by errors. A paper from Australia in 1995 showed similar findings confirming that harm to patients was more common than previously thought. ${ }^{7}$ These papers triggered thought as to how we could systematically improve patient safety in healthcare.

In the UK, the Department of Health reflected on how to take this forward. In their report An organisation with a memory, published in 2000, the development of 
'clinical governance' at a local level was seen as a road map to deliver better care. ${ }^{8}$ As a way of enhancing patient safety it called for unified reporting mechanisms, and a systems approach in preventing, analysing and learning from error. All of this was to be undertaken in the context of a more open culture in which lessons were embedded into an organisation's culture and practice. This was followed by the report Building a safer NHS for patients in 2001 and the establishment of The National Patient Safety Agency (NPSA) to help progress this work. ${ }^{9}$

In 2003, the Agency for Healthcare Research and Quality in the USA produced their four part patient safety initiative framework, while in the UK in 2004 the NPSA launched 'seven steps to patient safety', a guide to good practice intended to offer NHS organisations practical guidance and support on improving patient safety. ${ }^{10,11}$ The elements identified in these plans can be used to illustrate the mechanisms for the development of safer healthcare.

\section{STRATEGIES TO DEVELOP SAFER HEALTHCARE}

Strategy one: Identifying threats to patient safety by incident reporting. This is about identifying the errors and causes of patient injury associated with healthcare. This involves collecting relevant data where patient injury, or 'near misses' have occurred. An example already known to dentists is the 'yellow card scheme' for the reporting of adverse reactions to medicines, organised by the MHRA. ${ }^{12}$ Less well known to dentists is that the NPSA in England and Wales operates a national errorreporting system, the National Reporting and Learning System, to which anonymous reports of adverse events or 'near-misses' can be made. Hospital errors tend to be well represented as reports are uploaded to an online system from individual organisations' local risk management systems. The scheme sends healthcare providers regular patient safety alerts derived from analysing incident reports and other safety information. The use of the system is mandatory for reporting 'never events': a list of serious, preventable incidents such as leaving a foreign body inside the patient after surgery, which should never happen. Since 2010, reporting has also been mandatory for serious patient safety incidents - those leading to severe harm or death. The system is otherwise voluntary and a recent paper has highlighted the low volume of incidents reported by dentists in England and Wales. ${ }^{13}$

In Finland, a similar incident reporting system is available. Since 2007, utilisation of a voluntary and anonymous patient safety incident reporting system (HaiPro) has been in use but a recent survey indicates only $6 \%$ of the dentists who took part in the survey used the system. ${ }^{14}$ The majority of dentists either did not have access to or did not know of the system.

As dentists work in individual or small group practices they stand to gain most from a larger pooling of information regarding adverse patient safety issues offered by using such systems. Patient safety incident reporting systems appear to deserve further development in dentistry.

Strategy two: Evaluating incidents and identifying best practice. Hospitals have been at the forefront of operating incident reporting systems, to which all staff are encouraged to contribute. When incidences of patient harm or a 'near miss' occurs, the details are logged onto a database where the relative frequency of different types of incident and their relative severity can be analysed. From this information, efforts can be made to identify underlying causes and evidence-based safety practices can be implemented. Once identified, significant incidents can be analysed by various strategies including 'root cause analysis'. This is where the investigators systematically attempt to identify what happened, why it happened, and what can be done to stop or reduce the likelihood of it happening again. The 'lessons learnt' are then implemented into future practice.

Strategy three: Communication and education about patient safety. This is about recognising that healthcare has its risks and that we need to identify and reduce those risks to patient safety by effective communication, education, training and audit of practice. As a result of their undergraduate education, most dentists are already aware of what should be dental 'never events'. The inhalation of an endodontic file because a rubber dam or a parachute chain was not used, or the giving of penicillin to a penicillin allergic patient are two examples of 'never events'. In addition to established well known risks, new safety risks emerge as clinical developments occur. The MHRA drug safety update on new risks and cautions on medication use, arising from reports to the 'yellow card scheme', and subsequently incorporated into the British National Formulary, is an example known to many dentists. Further examples of guidance are given in the publications produced by the UK dental defence societies based on the complaints or litigation that the defence societies have dealt with. Such publications frequently offer analysis of cases received, and while giving guidance on how litigation maybe avoided in the future, also provide 'lessons learnt' on how patient safety can also be improved. In secondary dental care, many hospitals hold 'audit and clinical effectiveness' sessions where routine work is suspended to allow hospital teams to come together and discuss clinical effectiveness issues, including patient safety, to 'learn lessons' and give focus to improving quality of patient care.

Strategy four: Building a safety culture. This means a priority is given to patient safety and commitment to overall continuous improvement within the workplace. It requires an open culture in which incidents or service failure can be viewed as an opportunity to improve the service rather than as a source of blame. It requires a commitment to share our experiences and data, both good and bad, so that we can all learn from them. As with all of healthcare, dentistry depends on close teamwork and patient safety should be integral to dental team training and working. The ultimate aim is to create a culture that puts patient safety at the heart of what all healthcare professionals, including dentists and dental care professionals, do. The importance of culture is sometimes downplayed or overlooked, but as the recent Berwick Report noted; 'In the end, culture will trump rules, standards and control strategies every single time, and achieving a vastly safer NHS will depend far more on major cultural change than on a new regulatory regime. ${ }^{3}$

Recently several organisations, researchers and clinicians in various parts of the world have started to address the issue of patient safety in dentistry. ${ }^{1,4,5,13,14}$ There remains the capacity to improve patient safety by learning from each other and building a safety culture. Early research indicates that we have more to learn both in primary and secondary care dentistry. A recent study from Finland shows that wide variation in risk management practice exists between individual dentists and organisations. ${ }^{14}$ The Finnish authors acknowledge that no one strategy alone to improve patient safety is likely to be sufficient and improvements will depend on a combination of approaches.

In 2008, a commitment to making quality and safety the 'organising principle' of the NHS was made by the British Government in its white paper, High quality care for all. ${ }^{15}$ Despite this high profile initiative, improvement in the UK has been slow. The simple objective of avoiding preventable adverse events would seem straightforward but remains difficult to implement. Key challenges in implementing patient safety solutions in medicine have been identified including visibility, ambiguity, complexity and autonomy. ${ }^{16}$ The difficulties identified in 
medicine are just as true in dentistry as well, however, they should not act as a deterrent in the need to identify and mitigate patient safety problems. Several authors have made the point that education in patient safety issues should be considered for all dentists in undergraduate and postgraduate curricula to help raise awareness of this important issue. ${ }^{5,14}$

Standards for the dental team published by the General Dental Council (GDC) in the summer of 2013 makes several statements of relevance with respect to patient safety. ${ }^{17}$ Principle one (put patient's interests first) states in standard 1.5.4 'You must record all patient safety incidents and report them promptly to the appropriate national body' Principle eight (raise concerns of patients at risk) states in standard 8.1 'You must always put patients' safety first'. This brief overview has highlighted some of the strategies that have contributed to improving patient safety. Our challenge now is to continue to broaden our understanding of patient safety issues that matter in dentistry, develop strategies that reduce the risks for our patients and to develop a patient safety culture within our teams.

1. Council of European Dentists general meeting: CED resolution on patient safety. CED, 2008.

2. Mid Staffordshire NHS Foundation Trust. Report of the Mid Staffordshire NHS Foundation Trust public enquiry. London: The Stationary Office, 2013.

3. National Advisory Group on the Safety of Patients in England. A promise to learn - a commitment to act. London: Department of Health, 2013. Online report available at https://www.gov.uk/government/ uploads/system/uploads/attachment_data/ file/226703/Berwick_Report.pdf (accessed June 2014).

4. Ramoni R B, Walji M F, White J et al. From good to better. Toward a patient safety initiative in dentistry. JADA 2012; 143: 956-960.

5. Yamalik N, Perea-Perez B. Patient safety and dentistry: what do we need to know? Fundamentals of patient safety, the safety culture and implementation of patient safety measures in dental practice. Int Dent J 2012; 62: 189-196.

6. Brennan T A, Leape L L, Laired N M et al. Incidence of adverse events and negligence in hospitalized patients: results of the Harvard Medical Practice Study. N Eng/ J Med 1991; 324: 370-376.

7. Wilson R M, Runciman W B, Gibberd R W, Harrison B T, Newby L, Hamilton J D. The quality in australian health care study. Med J Aust 1995; 163: 458-471.

8. Department of Health. An organisation with a memory. London: The Stationary Office, 2000.

9. Department of Health. Building a safer NHS for patients. London: The Stationary Office, 2001.

10. Seven steps to patient safety. London: National Patient Safety Agency, 2004. Online steps available at http://www.npsa.nhs.uk/sevensteps (accessed June 2014).

11. Agency for Healthcare Research and Quality. $A H R Q$ 's patient safety initiative: building foundations, reducing risk. Interim report to the Senate Committee on appropriations. Rockville, Md: AHRQ, 2003. Online report available at http://www.ahrq.gov/qual/ pscongrpt/ (accessed June 2014).

12. Yip J, Radford D R, Brown D. How do dentists deal with adverse drug reaction reporting? Br Dent $J$ 2013; 214: E22.

13. Thusu S, Panesar S, Bedi R. Patient safety in dentistry - state of play as revealed by a national database of errors. Br Dent J 2012; 213: E3.

14. Hiivala N, Mussalo-Rauhamaa H, Murtomaa H. Patient safety incident prevention and management among Finnish dentists. Acta Odontol Scand 2013; 71: 1663-1670.

15. Darzi A. High quality care for all: NHS next stage review final report. London: The Stationary Office, 2008.

16. Leistikow I P, Kalkman CJ, de Bruijn H. Why patient safety is such a tough nut to crack. BMJ $2011 ; 342$ : d3447.

17. General Dental Council. Standards for the dental team. London: GDC, 2013. 